\title{
Internal perceptual system noise and redundancy in simultaneous inputs in form identification'
}

\author{
Charles W. Eriksen and Joseph S. Lappin \\ UNIVERSITY OF ILLINOIS
}

\begin{abstract}
Abstraet
Identification accuracy of forms was studied as a function of the number of simultaneous occurrences of the form on different foveal locations. A model for computing perceptual independence was presented and the data suggest that at a given moment in time internal noise for different elements in the visual perceptual system, represented by different foveal locations, is uncorrelated.
\end{abstract}

\section{Introduction}

A concept of internal perceptual noise has been applied fruitfully to problems in auditory (Swets, 1964) and visual perception (FitzHugh, 1957; Eijkman \& Vendrik, 1959). It accounts for the varying sensitivity of the $S$ from trial to trial when the signal is at constant energy level. In part, the concept is grounded in the electrophysiological evidence that neurons and ganglia show spontaneous discharges in the absence of external stimulation (FitzHugh, 1957). The temporal distribution of this internal noise has been studied by Eriksen \& Hoffman (1963) in visual perception, and the question arises as to whether in vision, at least, this internal noise may not have an areal as well as a temporal distribution. It would seem reasonable that at any given moment in time internal noise is different in different parts of the visual system as represented by different foveal locations. Closely adjacent areas, sharing essentially the same microenvironment, may be expected to show some noise correlation, but at some minimum separation noise becomes uncorrelated.

The possibility of such an areal distribution of internal noise can be tested by the following experimental arrangement. An exposure duration sufficient for an above-chance hit rate (HR) for identification of simple forms singly presented is obtained. Then, keeping exposure duration constant, two or more of the same form are presented in a single exposure separated by experimentally varied foveal distances. If the internal noise corresponding to the different foveal areas simultaneously stimulated in a single exposure is less than perfectly correlated, the S'S HR should increase with multiple occurrences of the same form. Further, when the retinal or foveal spacing is far enough so that the internal noise is completely uncorrelated, the gain in $\mathrm{HR}$ accruing through two, three, four, or more simultaneous exposures of the same form should be predictable from a model based on independence.

To determine whether a given retinal spacing leads to uncorrelated noise, a model is needed to predict what the increment in HR should be if the two areas are indeed independent or uncorrelated. The usual prob- ability formula for predicting the occurrence of one or the other or both of two independent events, $2 \mathrm{P}-\mathrm{P}^{2}$, where $P$ represents the HR for a single presentation, is inappropriate. A given HR contains not only the proportion of times the S correctly perceived the stimulus but also the number of times he guessed correctly. Since the $\mathrm{S}$ is allowed only one response following a multiple presentation of the same form in a single exposure, the above formula has the effect of giving double weight to the guessing component in the S's response.

A somewhat more appropriate model can be constructed by assuming that a given $\mathrm{HR}$ is composed from two states in the $\mathrm{S}$, state $\mathrm{A}$ where he perceives the stimulus and state $\mathrm{B}$ where he guesses. In a twocategory judgmental task where the a priori probability of a correct response is .5 , the proportion attributable to state A can be estimated by assuming that the $\mathrm{S}$ guessed correctly the same number of times he guessed incorrectly.

A more refined model is obtained if we take cognizance of the fact that a S's perceptual states are more complex than completely perceiving the stimulus or not perceiving it at all. Most Ss have no difficulty in reliably classifying their subjective confidence in their judgments into three categories; "certain," "think so," and "guess." These three ratings are typically found to have differential HRs. For judgments classed as "confident," HRs are typically higher than for judgments classified as "think so," and "guess" judgments frequently have a HR not much above chance. If we require an $\mathrm{S}$ to use three confidence categories for each judgment, we can make the assumption that these confidence ratings reflect different perceptual states: A, B, and C. The HR associated with each of these states can be empirically determined. The HR to be expected from two independent opportunities to perceive where each opportunity has the same $H R$, is obtained by first multiplying the two matrices,

$$
\begin{aligned}
& {\left[\begin{array}{l}
\mathrm{P}_{\mathrm{A}} \\
\mathrm{P}_{\mathrm{B}}
\end{array} \mid\left[\begin{array}{lll}
\mathrm{P}_{\mathrm{A}} & \mathrm{P}_{\mathrm{B}} & \mathrm{P}_{\mathrm{C}}
\end{array}\right]\right.} \\
& {\left[\begin{array}{l}
\mathrm{P}_{\mathrm{C}}
\end{array}\right]}
\end{aligned}
$$
Next all proportions containing a state A are added together and multiplied by the state A HR. All terms having a state $B$ but not a state $A$ are then added together and multiplied by the state B HR and finally the term $\mathrm{P}_{\mathrm{C}} \mathrm{P}_{\mathrm{C}}$ is multiplied by the state $\mathrm{C}$ HR. The summing of the three products then yields the HR to be expected for two independent opportunities to perceive. As can be seen the assumption has been made that 
when a combination of two different perceptual states occurs on a given trial the probability of a correct response is determined by the highest HR associated with the two perceptual states that are in combination.

The case where there are $n$ independent opportunities to perceive is computed according to the same logic. The probability that a perceptual state other than A will occur on all $\mathrm{n}$ independent opportunities to perceive is given by $\left(1-P_{A}\right)^{n}$. The probability that perceptual state $\mathrm{C}$ will obtain on all $\mathrm{n}$ independent opportunities is given

by $P_{C A}-\bar{B}=P_{C}{ }^{n}$. The probability that a $B$ state will occur but not an $\mathrm{A}$ in the $\mathrm{n}$ opportunities is $\mathrm{P}_{\mathrm{BA}}=\left(1-\mathrm{P}_{A}\right)^{n}-\mathrm{P}_{C}{ }^{\mathrm{n}}$. Finally, the probability of an $\mathrm{A}$ state occurring on one or more of the independent opportunities is given by $P_{A v A}=1-P_{B A}-P_{C}{ }^{n}$.

\section{Method}

The form stimuli were the capital letters A, T, and U, black on white and subtending $.3^{\circ}$ of visual angle. They were presented in a tachistoscope containing a dark adaptation field with a faintly glowing $\mathrm{x}$ fixation point. Three retinal spacings of the stimuli were employed. For the $1 / 2^{\circ}$ condition the stimuli occurred on the circumference of an imaginary circle having a radius of $1 / 2^{\circ}$ of visual angle around the fixation point. For the $1^{\mathrm{O}}$ and $2^{\mathrm{O}}$ conditions the circumference of this imaginary circle subtended $1^{\circ}$ and $2^{\circ}$ radii from the center of the fixation point. During several practice sessions an exposure duration was determined for each $\mathrm{S}$ that resulted in 50 to $55 \%$ identification accuracy for only a single presentation of a form at the given retinal spacing. Each $\mathrm{S}$ engaged in four sessions at each of the three retinal spacings. During each session trials containing one, two, four and six simultaneous occurrences of the same form were randomly presented to him for judgment. The forms appeared in a random arrangement in the positions on the imaginary circle that would correspond to the numbers two, four, six, eight, ten and twelve on a clock. Thus on some trials a $\mathrm{S}$ would be presented a single $\mathrm{A}$ in one of these locations. On other trials two, four or six As would be simultaneously presented in these positions. Similarly, for the Ts and Us. In addition to making a forced-choice judgment as to which of the three forms had been presented on a given trial, the $\mathrm{S}$ also rated his subjective confidence in his judgment using the numbers 1,2 or 3 to reflect respectively, "confident," "think so," and "guess." Ss were counterbalanced through all three retinal separation conditions for a total of 12 experimental sessions per S. During each session each $\mathrm{S}$ made 18 judgments for single stimulus occurrences and the same number of judgments for each of the two, four and six stimulus presentations for a total of 72 judgments per session.

The Ss were six practiced Os recruited from graduate students and were paid for their services.

\section{Results and Diseussion}

Percent correct identifications were analyzed in a three-way analysis of variance (Ss, retinal separation, and number of repetitions of the form in a single presentation). The effect of the number of form repetitions was highly significant (F $3 / 15=42.5)$ and a significant condition by Ss effect was also obtained. This latter, however, is attributable to our inability to match Ss across conditions at an exposure level yielding exactly $50 \%$ identification accuracy. There was, however, no indication of an interaction between number of stimuli presented in an exposure and degree of retinal separation (F $6 / 30=1.18$ ).

The data have been pooled through retinal separation conditions in Fig. 1 where the $\pm 2 \sigma$ range is indicated

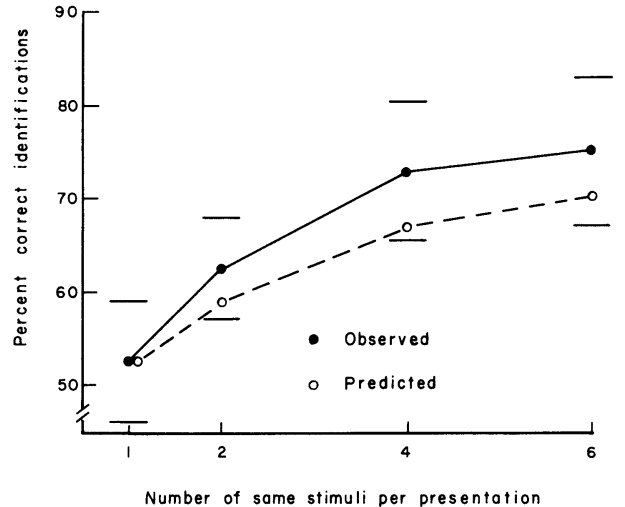

Fig. 1. Predicted and obtained form identification as a function of the number of simultaneous occurrences of the form.

for the obtained values. Identification accuracy increases in a negatively accelerated fashion with an increase in the number of repetitions of the same form in a single exposure. The dotted line curve in Fig. 1 is the predicted identification accuracy or HR using the model outlined in the introduction and the data obtained by pooling through Ss for the condition where only one stimulus was presented in an exposure. The probability of perceptual states A, B and C, as inferred from the Ss' confidence ratings, were $.117, .291$, and .592 . The differential HRs associated with these perceptual states were, respectively, .812, .597 , and .437 .

The model provides a satisfactory fit for the observed data points. It may also be pointed out that the predictions are made on the basis of six empirically derived values for each $\mathrm{S}$, each prone to sampling, error. Whatever error occurs in estimating the probabilities and HR for the three perceptual states in the single presentation condition becomes magnified as $n$ increases. The lack of a significant interaction between degree of retinal spacing of the stimuli and number of stimuli presented in a trial suggests that, at the degree of separation between forms employed in this study, the internal perceptual noise corresponding to the respective foveal areas stimulated is already uncorrelated. The lack of correlation is attested by the close fit of the observed data points to the points predicted on the assumption of zero noise correlation. Even under the $1 / 2^{\mathrm{O}}$ condition the forms were about $1^{\mathrm{O}}$ apart a large proportion of the time due to their spacing on the $1 / 2^{\mathrm{O}}$ radius.

\section{References}

EIJKMAN, E., \& VENDRIK, A. J. H. Detection theory applied to the absolute sensitivity of sensory systems. In J.A. Swets (Ed.), Signal detection and recognition by human observers. New York: Wiley \& Sons, 1964. Pp. 392-410。

ERIKSEN, C. W., \& HOFFMAN, M. Form recognition at brief durations as a function of adapting field and interval between stimulations. J. exp. Psychol., 1963, 66, 485-499.

FITZHUGH, R. The statistical detection of threshold signals in the retina. J. gen. Physiol., 1957, 40, 925-948.

SWETS, J. A. (Ed.) Signal detection and recognition by human observers. New York: Wiley \& Sons, 1964.

Note

1. This investigation was supported by Public Health Service research grant MH-1206 and Public Health Service research career program award K6-MH-22,014. 